\title{
Nonmotor Regions Encode Path-Related Information During Movements
}

\author{
Macauley S. Breault, Pierre Sacré, Jacob J. Johnson, Matthew Kerr, Matthew D. Johnson, Juan Bulacio, Jorge \\ González-Martínez and Sridevi V. Sarma - Member, IEEE, John T. Gale
}

\begin{abstract}
Sensorimotor control and the involvement of motor brain regions has been extensively studied, but the role nonmotor brain regions play during movements has been overlooked. This is particularly due to the difficulty of recording from multiple regions in the brain during motor control. In this study, we utilize stereoelectroencephalography (SEEG) recording techniques to explore the role nonmotor brain areas have on the way we move. Nine humans were implanted with SEEG depth electrodes for clinical purposes, which rendered access to local field potential (LFP) activity in deep and peripheral nonmotor structures. Participants performed fast and slow arm reaching movements using a robotic manipulandum. In this study, we explored whether neural activity in a given nonmotor brain structure correlated to movement path metrics including: path length, path deviation, and path speed. Statistical analysis revealed correlations between averaged neural activity in middle temporal gyrus, supramarginal gyrus, and fusiform gyrus and our path metrics both within and across the subjects. Furthermore, we split trials across subjects into two groups: one group consisted of trials with high values of each path metric and the other with low values. We then found significant differences in LFP power in specific frequency bands (e.g. beta) during movement between each group. These results suggest that nonmotor regions may dynamically encode path-related information during movement.
\end{abstract}

\section{INTRODUCTION}

First mapped in 1917, recordings of cortical motor regions have been valued for their explicit involvement in movement execution, both in human and nonhuman primates $[1,2]$. However, successful motor control relies on communication between motor and nonmotor areas, such as integrating sensory information to update the movement trajectory or to attenuate movement or to respond to unexpected perturbations [3-6]. Lesion studies and nonhuman primate studies have shown that nonmotor regions play an important role in movement but remain largely undocumented $[7,8]$. However, these regions could prove critical for future brain-machine interface applications for subjects whose motor areas are too impaired to provide reliable information.

What makes nonmotor regions studies in humans particularly limited is the difficulty in recording from these regions during strenuous movement tasks. Previous studies of these regions have relied on fMRI, ECoG, and MEG to record in humans, each suffering from spatial or temporal resolution issues in one way or another $[9,10]$. The cumbersome set-ups required to perform natural movements also proves problematic within confined spaces [11].

In this study, we exploit a unique experimental set-up wherein we gain access to brain areas in humans not previously studied while executing a motor task. SEEG allows for the recording of multiple brain structures simultaneously with millisecond temporal resolution [11]. This technique has been previously used to correlate highfrequency activity (HFA) to responses of movement perturbations [12].

Here we examined the correlation between behavioral features and nonmotor areas to investigate whether these regions encode aspects related to the way we move. In this study, epileptic patients implanted with electrodes for clinical purposes performed center-out reaching task using a robotic arm under instructed speed conditions. Upon behavioral analysis, movements were broken down into three basic path features (path length, path deviation, and path speed). These path features were found to correlate with neural activity in nonmotor areas in specific frequency bands (e.g. beta), including the supramarginal gyrus, middle temporal gyrus (MTG), and fusiform gyrus, both within and across the subjects. These results suggest that nonmotor regions may encode static, path-related information during movement.

\section{II.METHODS}

\section{A. Subjects}

SEEG recordings were performed in medically refractory epileptic patients for the clinical purpose of finding the epileptogenic zone for possible resection. This study did not alter any invasive procedure as electrode location was made independently of the previous study based on pre-operative measures. Subject enrollment was completely voluntarily and all subjects gave informed consent. Experimental protocols were approved by the Cleveland Clinic Institutional Review Board. Methods were carried out in accordance with approved guidelines. Criteria allowed for 9 individuals over the age of 18 with the ability to provide

M. S. Breault, P. Sacré, and S. V. Sarma are with the Department of Biomedical Engineering at Johns Hopkins University, Baltimore, MD 21218 USA (email: mbreaul1@ jhu.edu,).

J. J. Johnson is with the Department of Electrical and Electronics Engineering at Indian Institute of Technology Guwahati, Assam 780139, India (e-mail: j.johnson@iitg.ernet.in).

M. Kerr is with the Department of Engineering at University of Cambridge, Cambridge CB2 1PZ UK.

M. D. Johnson is with the National Institute of Neurological Disorders and Stroke at National Institutes for Health, Bethesda, MD 20892 USA.

J. Bulacio is with the Department of Neurology and the Epilepsy Center at Cleveland Clinic, Cleveland, OH 44195 USA.

J. González-Martínez is with the Department of Neurological Surgery and the Epilepsy Center at Cleveland Clinic, Cleveland, OH 44195 USA.

J. T. Gale is with the Department of Neurosurgery at Emory University, Atlanta, Georgia 30322 USA. (e-mail: john.t.gale@emory.edu) 
informed consent and to perform the behavioral task. Alterations were not made to their clinical care other than the behavioral experiments.

\section{B. Stereoelectroencephalographic (SEEG) Implantations}

The SEEG depth electrodes (PMT Corporation, MN, USA) were implanted at the Cleveland Clinic using coregistered three-dimensional CT and MRI scans [11].

Approximately 8 to 13 stereotactically placed electrodes were implanted per subjects. Each electrode contact had a $0.8 \mathrm{~mm}$ diameter, was $2 \mathrm{~mm}$ long, and contacts were spaced $1.5 \mathrm{~mm}$ apart. Depth electrodes were inserted using a robotic surgical implantation platform, (ROSA, Medtech Surgical Inc., USA) in either orthogonal or oblique orientation. This allowed for intracranial recording from lateral, intermediate and/or deep cortical and subcortical structures in a threedimensional arrangement [13]. The day prior to surgery, volumetric pre-operative MRIs (T1, contrasted with Multihance ${ }^{\circledR}-0.1 \mathrm{mmol} / \mathrm{Kg}$ ) were obtained and used to preoperative plan safe electrodes trajectories. Insertion trajectories were adjusted to avoid vascular structures. Electrode contacts were labeled according to the anatomical location from post-operative images with the agreement of two clinical experts.

\section{Electrophysiological Recordings}

Neural recordings were collected onsite using a clinical electrophysiology acquiring system activity (Nihon Kohden 1200, Nihon Kohden America, USA) in the Epilepsy Monitoring unit at a sampling rate of $2 \mathrm{KHz}$ from peripheral to deep brain structures. Recording sessions for this study were absent of epileptic activity.

\section{Behavioral Task}

Subjects $(n=9)$ performed a speed and goal-directed reaching movements using a robotic manipulandum from the InMotion ARM Interactive Therapy System (Interactive Motion Technologies, Watertown, MA, USA) to move the cursor to complete the behavioral task displayed on a monitor as previously described (Fig. 1 (top)) [12,14,15]. This system allowed for precise tracking of arm movements over a horizontal plane as the subjects manipulated a cursor presented on the screen along with the task stimuli. The behavioral task was prepared with a MATLAB ${ }^{\circledR}$ interface (Mathworks, Natick, MA) using MonkeyLogic [16,17].

The overview of the behavioral task was to move and maintain the cursor from the center to a target within an instructed fast or slow time. Speeds were relative to the subject based on calibrated trials of their fastest movements prior to task initiation. A successful fast speed required a trial time between $60-80 \%$ of their calibrated speed while a slow speed required $20-40 \%$ of their calibrated speed.

Visual stimuli and task details are explained in Fig. 1 (bottom). Trials begin with speed instruction, visualized by a vertical bar. The top or bottom portion would be highlighted green to indicate the instructed speed of fast or slow, respectively. After fixation by means of moving the cursor into the center, the target of the trial is displayed prior to movement for $2 \pm 0.25$ seconds. Go cue initiates when the target turns green after the delay. Movement onset is triggered when the subject's cursor leaves the center. The

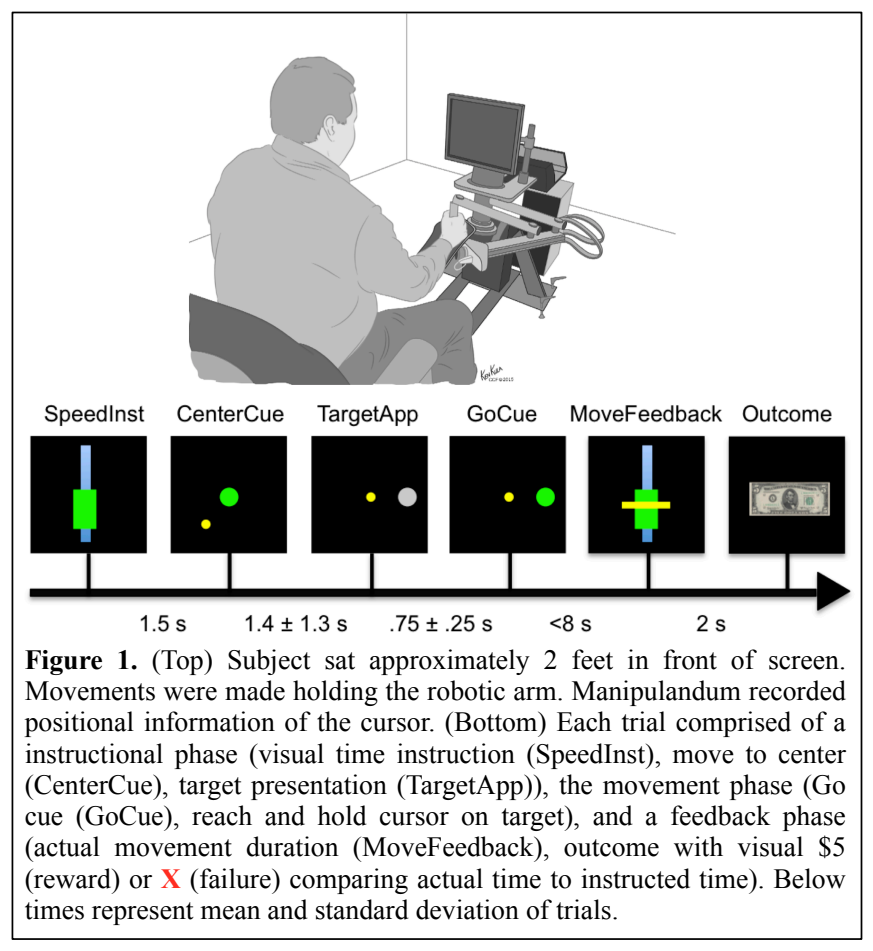

subject is expected to move their cursor into the target within 8 seconds including 0.5 seconds of holding. After successful holding, speed feedback is provided comparing the subject's trial speed to the instructed speed on the speed bar for 2 seconds. The outcome is revealed as a visual field of either a $\$ 5$ bill upon success or a red $\mathrm{X}$ upon failure based on their performance.

\section{E. Behavioral Analysis}

All electrophysiological and behavioral analyses were conducted offline using custom MATLAB scripts. Behavioral analysis was performed on positional data between movement onset and the first hit in the target during the movement phase of unperturbed trials. Positional data were smoothed with a low-pass Butterworth filter (cutoff frequency of $10 \mathrm{~Hz}$ ).

Three metrics were chosen to capture key features of movements on a trial-by-trail basis. These uncorrelated metrics are path length, path deviation, and path speed. Path length is the arc length normalized by the length of the straight line between the point where they left the center and where they entered the target [18]. Path deviation measures the average deviation each sample point is away from the task axis, defined as the straight line from the center of fixation to the target [19]. Path speed is the distance covered in the trial divided by the time of the trial. This metric was used by the task program to evaluate trial speed compared to the instructed speed for each session. All metrics were normalized for each subject.

\section{F. Neural Data Analysis}

Neuronal activity collected from SEEG electrodes are analyzed using frequency analysis on the voltage data. The $\log$ power spectrum is cut into 10 sections with widths of 10 $\mathrm{Hz}$. The z-scored of the log power is calculated from the distribution of the log power per section over the entire 

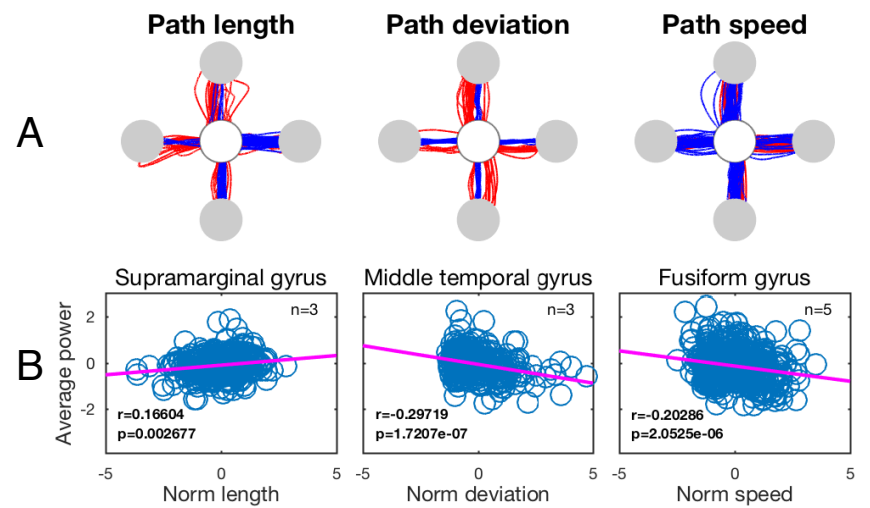

C
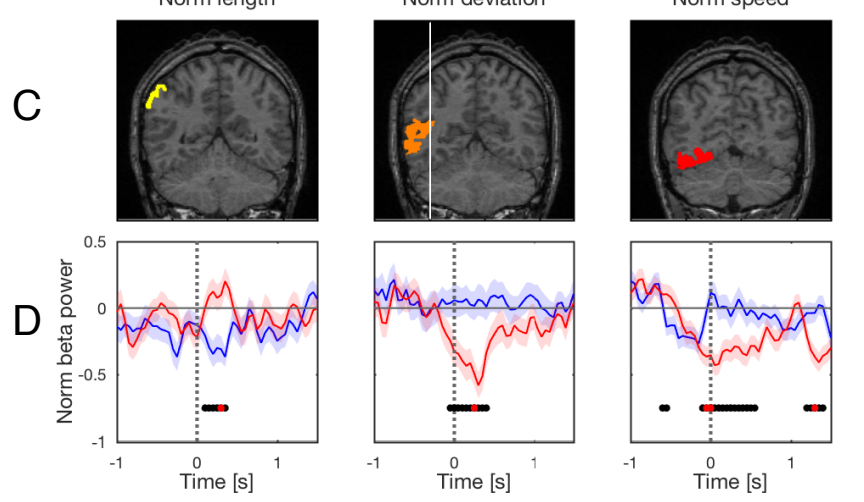

Figure 2. Example of a complete population analysis for each metric. Columns represent the path metric and rows associates to corresponding description. (A) Raw paths overlaid with groups where high metric values are red and low metric values are blue. (B) Population scatter plot of average beta power and metric normalized to the subject with least square line (magenta) to show trend. Number of subjects, correlation coefficient, and associated p-value also shown. (C) Coronal view of brain MRI with areas of interest highlighted for demonstrative purposes. (D) Average normalized beta power plotted over time for two groups, with a window of $[-1,1.5]$ seconds time locked to movement onset at 0 seconds. Average population trial completion was about 1 second after movement onset. The colors are consistent with previous representations. The shaded area shows for one standard error. The dots under the time series shows the results of the clustering analysis, where red dots signify a significant difference between the two groups.

session time with the top and bottom 5 percentile excluded for artifact rejection.

To capture a form of neural spiking from the data, three frequency bands were chosen: beta $(15-30 \mathrm{~Hz})$, gamma $(30-100 \mathrm{~Hz})$, high gamma $(100-200 \mathrm{~Hz})$. Each band contained approximately ten frequency bins. The z-scored $\log$ power was averaged over these bins across bands to obtain a temporal representation of neuronal firing. For trialby-trial correlation analysis, mean band power was averaged between movement onset and first target hit to obtain one number per trial for each subject.

\section{G. Subject Specific Analysis}

To investigate if path-related information was being encoded by any nonmotor brain regions, a static analysis was devised. This comprised of correlating the average neural activity in each band to each path metric on a trial-bytrial basis within each subject. The Pearson correlation coefficient and corresponding p-value were calculated. This was repeated for each condition (band, metric, subject).

Conditions with significant correlations $(p<0.05)$ were split into two groups: one group consisted of the top $20 \%$ of trials with the highest values and the other with the bottom $20 \%$ of trials with the lowest values. These trials were temporally plotted against each other time locked at movement onset and a nonparametric cluster analysis was performed between these two conditions [20]. Conditions with a significant difference $(p<0.05)$ in neural activity were extracted and deemed as encoding path-related information.

\section{H. Population Analysis}

To make a general statement about neural encoding, common conditions must be observed across three or more sessions. Therefore, the trial-average analysis described above was repeated on brain regions that were significant in both correlation and temporal analysis as well as observed in at least three sessions. The combined trials were separated into groups by high and low values within each path metric, relative to the subject. Again, correlation coefficients were calculated and a nonparametric cluster analysis was performed on the separated groups.

\section{RESULTS}

Multiple nonmotor regions correlated with path-related metrics. Here we examine brain areas that were found to encode each metric according to the population analysis.

\section{A. Path analysis}

Since metrics were calculated relative to each subject, subject performance varied greatly; e.g. what was a fast trial for one subject may have been considered a slow trial for another. Fig. 2A demonstrates the ability of the metrics to represent different types of paths, where red paths indicate trials with high metric values and blue paths represent trials with low values. Path length separates direct paths (blue) from indirect paths (red) to the target. Path deviation separates paths that were inline with the task axis (blue) from paths that deviated from the target (red). Path speed meant to separate trials with a slow time (blue) from fast trials (red). Though the metric inherently does not directly related to visual path characteristics, slow trials appear to be a combination of wavier or have larger path lengths than fast trials.

\section{B. Population correlations}

Many regions were found to be correlated with path information but only a select few were common across subjects. All regions selected had a significant correlation with the metric $(p<0.05)$ with the highest correlation coefficients amongst other regions. These regions were supramarginal gyrus for path length, MTG for path deviation, and fusiform gyrus for path speed. Fig. 2C shows the scatter plots with the associated correlation values per metric. Supramarginal gyrus has a positive correlation with path length, whereas middle temporal gyrus and fusiform gyrus have negative correlations with path deviation and speed, respectively. Fig. 2B shows the anatomical location of the brain regions, highlighted in an MRI slice.

\section{Population average power plots}

Shown in Fig. 2D are the normalized beta band power temporally averaged for each metric and associated brain area time locked to movement onset. The signs of the correlations in Fig. 2C are exhibited in the average power 
plots. Negatively correlated conditions, (MTG, path deviation) and (fusiform gyrus, path speed), appear to have a decrease in neural activity after movement onset for trials with high valued metrics and conversely for positively correlated conditions (supramarginal gyrus, path length).

\section{DISCUSSION}

Our results suggest that neural activity in nonmotor regions is associated with observed behavioral patterns through metrics that characterize path execution. This suggests that these nonmotor areas may be encoding information about the movement that was not previously known. Overall, beta band activity was more commonly correlated with the path metrics than gamma or high gamma activity. Beta band is known to become suppressed after movement onset and has been correlated with postmovements error [18]. This is similar to our observations with path deviation, a type of error, and MTG with suppressed beta sustained during the trials.

Path length: The supramarginal gyrus, a division of the inferior parietal lobe, has been previously imaged to be involved with motor attention [7,21]. The results from the population analysis indicate that trials with longer paths have an increase in neural activity that is delayed towards the end of the trial (Fig. 2D left). This suggests that longer trials may require more attention to motor control and hence more recruitment of neuronal firing at the end of the movement.

Path deviation: The MTG has been identified as apart of the visual processing known as V5, which has been shown to perceive and direct attention to motion in nonhuman primates and in humans [22,23]. It has also been shown in fMRI studies to correlate with distance along with the cingulate cortex (not shown) [24]. The negative correlation between path deviation and MTG produces a sharp suppression in neural activity for trials with large deviations (Fig. 2D center). This suggests that the MTG could be reacting to the movement deviation, perhaps due to the extra distance covered or attenuation required to complete drifted paths.

Path speed: Fusiform gyrus has been proposed to be associated with color processing, recognition, and spatial attention $[23,25,26]$. In Fig. 2D right, initially there is a decrease in activity for both fast and slow trials followed by a return to baseline for slow trials and a sustained suppression in fast trials until trial completion around 1 second. Based on our results, this difference in activity could be observing a form of selectivity that distinguishes between the motion being fast or slow. In this case, it is selective towards fast speeds through suppression whereas slow speeds return to baseline.

\section{ACKNOWLEDGEMENT}

This work was supported by a National Science Foundation grant (EFRI-MC3: \# 1137237) awarded to S.V.S., J.A.G., J.B., and J.T.G. and the ARCS Foundation awarded to M.S.B.

\section{REFERENCES}

[1] A. S. F. Leyton and C. S. Sherrington, "Observations on the excitable cortex of the chimpanzee, orangutan and gorilla," Exp. Physiol, vol. 11, no. 2, pp. 135-222, 1917.

[2] W. Penfield and E. Boldrey, "Somatic motor and sensory representation in the cerebral cortex of man as studied by electrical stimulation," Brain: A Journal of Neurology, vol. 60, pp. 389-443, Dec. 1937.

[3] M. Corbetta and G. L. Shulman, "Control of goal-directed and stimulusdriven attention in the brain," Nature Reviews Neuroscience, vol. 3, no. 3, pp. 201-215, Mar. 2002.

[4] M. Desmurget and S. Grafton, "Forward modeling allows feedback control for fast reaching movements," Trends in Cognitive Sciences, vol 4, no. 11, pp. 423-431, Nov. 2000.

[5] R. A. Andersen and C. A. Buneo, "Intentional maps in posterior parietal cortex," Annual Review of Neuroscience, vol. 25, no. 1, pp. 189-220, Mar. 2002.

[6] M. Kerr, et al., "The Role of Associative Cortices and Hippocampus during Movement Perturbations," Front. Neural Circuits, vol. 11, p. 26, April 2017.

[7] R. J. Perry and S. Zeki, "The neurology of saccades and covert shifts in spatial attention," Brain, vol. 123, no. 11, pp. 2273-2288, Nov. 2000.

[8] T. Aflalo, et al., "Decoding motor imagery from the posterior parietal cortex of a tetraplegic human," Science, vol. 348, no. 6237, pp. 906910, May 2015.

[9] N. K. Logothetis, "What we can do and what we cannot do with fMRI," Nature, vol. 453, no. 7197, pp. 869-878, June 2008.

[10] J. Diedrichsen, et al., "Neural correlates of reach errors," Journal of Neuroscience, vol. 25, no. 43, pp. 9919-9931, Oct. 2005.

[11] J. González-Martínez, et al., "Technique, results, and complications related to robot-assisted stereoelectroencephalography," Neurosurgery, vol. 78, no. 2, pp. 169-180, 2016.

[12] M. S. D. Kerr, et al., "High frequency activity correlates of robust movement in humans," in Proc. 36th Annu. IEEE EMBC, Chicago, 2014, pp. 4391-4394.

[13] J. González-Martínez, et al., "Robotic Epilepsy Surgery: Technique, Results and Complications Related to Robotic Assisted SEEG," Neurosurgery, vol. 78, no. 2, pp. 169-179, 2016.

[14] M. A. Johnson, et al., "Performing behavioral tasks in subjects with intracranial electrodes," J. Vis. Exp., no. 92, 2014.

[15] P. Sacré, et al., "Lucky rhythms in orbitofrontal cortex bias gambling decisions in humans," Scientific Reports, vol. 6, 36206, 2016.

[16] W. F. Asaad and E. N. Eskandar, "A flexible software tool for temporally-precise behavioral control in MATLAB," Journal of Neuroscience Methods, vol. 174, no. 2, pp. 245-258, Sep. 2008.

[17] W. F. Asaad, et al., "High-performance execution of psychophysical tasks with complex visual stimuli in MATLAB," Journal of neurophysiology, vol. 109, no. 1, pp. 249-260, Jan. 2013.

[18] H. Tan, N. Jenkinson, and P. Brown, "Dynamic neural correlates of motor error monitoring and adaptation during trial-to-trial learning," $J$ Neurosci., vol. 34, no. 16, pp. 5678-5688, Apr. 2014.

[19] S. I. MacKenzie, T. Kauppinen, and M. Silfverberg, "Accuracy measures for evaluating computer pointing devices," in Proc. of the SIGCHI conference on Human factors in computing systems, Seattle, 2001, pp. 9-16.

[20] E. Maris and R. Oostenveld, "Nonparametric statistical testing of EEG and MEG-data," J. Neurosci. Methods, vol. 164, no. 1, pp. 177-190, 2007.

[21] M. F. S. Rushworth, Matthew, A. Ellison, and V. Walsh, "Complementary localization and lateralization of orienting and motor attention," Nature neuroscience, vol. 4, no. 6, pp. 656-661, 2001.

[22] E. P. Simoncelli and D. J. Heeger, "A model of neuronal responses in visual area MT," Vision research, vol. 38, no. 5, pp. 743-761, 1998.

[23] M. Corbetta, et al., "Attentional modulation of neural processing of shape, color, and velocity in humans," Science, vol. 248, no. 4962, p. $1556,1990$.

[24] P. Pinel, et al., "Modulation of parietal activation by semantic distance in a number comparison task," Neuroimage, vol. 14, no. 5, pp. 10131026, 2001.

[25] F. Castelli, et al., "Movement and mind: a functional imaging study of perception and interpretation of complex intentional movement patterns," Neuroimage, vol. 12, no. 3, pp. 314-325, 2000.

[26] L. Michels, et al., "Brain activity for peripheral biological motion in the posterior superior temporal gyrus and the fusiform gyrus: Dependence on visual hemifield and view orientation," Neuroimage, vol. 45, no. 1, pp. 151-159, 2009. 